\title{
Existence Theorems for Lagrange Control Problems with Unbounded Time Domain ${ }^{1}$
}

\author{
R. F. BAUM ${ }^{2}$ \\ Communicated by L. Cesari
}

\begin{abstract}
Existence theorems are proved for usual Lagrange control systems, in which the time domain is unbounded. As usual in Lagrange problems, the cost functional is an improper integral, the state equation is a system of ordinary differential equations, with assigned boundary conditions, and constraints may be imposed on the values of the state and control variables. It is shown that the boundary conditions at infinity require a particular analysis. Problems of this form can be found in econometrics (e.g., infinite-horizon economic models) and operations research (e.g., search problems).
\end{abstract}

Key Words. Existence theorems, Lagrange control problems, infinite horizon, boundary controls, conditions at infinity, search problems.

\section{Introduction}

In the present paper, we prove existence theorems for usual Lagrange control systems, in which the time domain is unbounded. For example, we discuss the existence of pairs $x(t)$ (trajectory), $u(t)$ (control), $x(t)$ absolutely continuous with values in $E_{n}, u(t)$ measurable with values in $E_{m}$, which minimize the cost functional

$$
I[x, u]=\int_{t_{1}}^{\infty} f_{0}(t, x(t), u(t)) d t
$$

and satisfy the state equation

$$
d x(t) / d t=f(t, x(t), u(t)), \quad t_{1} \leq t<\infty,
$$

\footnotetext{
${ }^{1}$ The author wishes to thank Professor L. Cesari for his many helpful comments and assistance in the preparation of this paper. This work was sponsored by the United States Air Force under Grants Nos. AF-AFOSR-69-1767-A and AFOSR-69-1662.

${ }^{2}$ Assistant Professor, Department of Industrial and Operations Engineering, University of Michigan, Ann Arbor, Michigan.
} 
with constraints

$$
(t, x(t)) \in A \subset E_{n}, \quad u(t) \in U(t, x(t)) \subset E_{m}, \quad t_{1} \leq t<\infty
$$

and boundary conditions

$$
\left(t_{1}, x\left(t_{1}\right), \lim _{t \rightarrow \infty} x(t)\right) \in B \subset E_{1+2 n} .
$$

Problems of this precise form can be found in econometrics (e.g., infinitehorizon economic models of the form of Examples 7.4 and 7.5) and in operations research (e.g., search problems, as in Examples 7.1 through 7.3).

The methods used here are extensions of those used by Cesari (Refs. 1-4), with the difference that the topology used is that of uniform convergence of the state functions on compact sets (compact open topology), as suggested by Cesari in Ref. 2, instead of the usual uniform topology (or $\rho$-metric). Boundary conditions at finite points can be framed in this approach, as usual. But, in this paper, we take into consideration also boundary conditions at infinity, as for instance

$$
x(-\infty)=0 \quad \text { or } x(+\infty)=1,
$$

conditions which are particularly difficult to handle in the open compact topology. However, in the present paper, we discuss also conditions at infinity, and corresponding existence theorems are given.

In Section 2, we formally present our control systems; and, in Section 3, we introduce some preliminary definitions. In Section 4, we state closure theorems for these systems, and in these theorems we stress only properties which are preserved in the passage to the limit in the compact open topology on the state variables. In Section 5, we introduce the concept of boundary conditions, together with suitable notations and conventions. Here, we take into consideration not only boundary conditions at finite times, but also boundary conditions at infinity. As mentioned, boundary conditions at infinity are, in general, not preserved in the compact open topology. Nevertheless, this occurs under hypotheses which will be stated in Section 5 . With this understanding, a closed class $\Omega$ of admissible pairs is one for which the boundary conditions (at finite times or at infinity) are preserved in the compact open topology. In Section 6, we then prove existence theorems for optimal solutions in closed classes $\Omega$. Finally, in Section 7 , we consider some applications of our results.

Free problems of the calculus of variations on infinite intervals, which depend on first derivatives or on higher derivatives, can be written in the Lagrange form above. Cinquini (Refs. 5-6), Faedo (Refs. 7-9), and others have given existence theorems for free problems, but they have not considered problems involving boundary conditions at infinity. We consider certain free problems in Section 7. 


\section{Description of the System}

We consider here control systems defined over a (possibly unbounded) time domain. Let $A$, the constraint set, be a fixed closed subset of the $t x$-space $E_{1} \times E_{n}$, with $t$ in $E_{1}$, and $x=\left(x^{1}, \ldots, x^{n}\right)$, the state variable, in $E_{n}$. For each $(t, x)$ in $A$, let $U(t, x)$, the control set, be a subset of the $u$-space $E_{m}$, $u=\left(u^{1}, \ldots, u^{m}\right)$ the control variable. Let

$$
M=\{(t, x, u):(t, x) \in A, u \in U(t, x)\},
$$

and let

$$
f(t, x, u)=\left(f_{1}(t, x, u), \ldots, f_{n}(t, x, u)\right)
$$

be a continuous vector function from $M$ into $E_{n}$. Let $B$, the boundary set, be a given closed subset of $E_{2 n+2}$.

We shall say that a pair $x(t), u(t), t_{1} \leq t \leq t_{2}$ (where, if $t_{1}$ or $t_{2}$ or both are infinite, we understand $-\infty<t<\infty, t_{1} \leq t<\infty$, etc.) is admissible for the system if $x(t)$ (trajectory) and $u(t)$ (control) satisfy the following conditions:

(a) $x(t)$ is absolutely continuous (AC) in every bounded interval of $t_{1} \leq t \leq t_{2}$

(b) $u(t)$ is measurable in $t_{1} \leq t \leq t_{2}$;

(c) $(t, x(t)) \in A$ for every $t_{1} \leq t \leq t_{2}$,

(d) $u(t) \in U(t, x(t))$ a.e. in $t_{1} \leq t \leq t_{2}$;

(e) $d x(t) / d t f\left(t, x(t), u(t)\right.$ a.e. in $t_{1} \leq t \leq t_{2}$.

For admissible pairs $x, u$, we take the cost functional $I[x, u]$ to be the (possibly improper) integral

$$
I[x, u]=\int_{t_{1}}^{t_{2}} f_{0}(t, x, u) d t
$$

$f_{0}$ a continuous function from $M$ into $E_{1}$.

We seek the absolute minimum of $I[x, u]$ in a suitable class $\Omega$ of admissible pairs. If $\tilde{x}, \tilde{u}$ has the property that

$$
I[\tilde{x}, \tilde{u}] \leq I[x, u]
$$

for all $x, u$ in $\Omega$, then we say that $\tilde{x}, \tilde{u}$ is an optimal pair, and we may say that $\tilde{u}$ is an optimal control and $\tilde{x}$ is an optimal trajectory.

\section{Preliminaries}

We shall need certain properties of set functions throughout the following. Given any set $F$ in a linear space $E$, we shall denote by $\mathrm{cl} F$ and co $F$ the closure of $F$ and the convex hull of $F$, respectively. 
For every $\left(t_{0}, x_{0}\right) \in A$, and $\delta>0$, let $N_{\delta}\left(t_{0}, x_{0}\right)$ denote the closed $\delta$ neighborhood of radius $\delta$ in $A$, that is, the set of all $(t, x) \in A$ at a distance $\leq \delta$ from $\left(t_{0}, x_{0}\right)$.

Let $F(t, x)$ be a variable set in Euclidean space $E$, a set function of $(t, x)$ in $A$. We shall say that $F$ is an upper semicontinuous function of $(t, x)$ at the point $\left(t_{0}, x_{0}\right)$ in $A$ if, given $\epsilon>0$, there is a $\delta=\delta\left(t_{0}, x_{0}, \epsilon\right)>0$ such that $(t, x) \in N_{\delta}\left(t_{0}, x_{0}\right)$ implies

$$
F(t, x) \subset\left[F\left(t_{0}, x_{0}\right)\right]_{\epsilon},
$$

where $[F]_{\epsilon}$ denotes the closed $\epsilon$-neighborhood of $F$ in $E$. If $F(t, x)$ is an upper semicontinuous function for all $(t, x) \in A$, then we shall say that $F(t, x)$ is an upper semicontinuous function in $A$.

Again, let $F(t, x),(t, x)$ in $A$, be a variable set in $E$. For every $\delta>0$, let

$$
F\left(t_{0}, x_{0} ; \delta\right)=\cup F(t, x),
$$

where the union is taken for all $(t, x) \in N_{\delta}\left(t_{0}, x_{0}\right)$. We shall say that $F(t, x)$ has property $(\mathrm{U})$ at $\left(t_{0}, x_{0}\right)$ in $A$ if

$$
F\left(t_{0}, x_{0}\right)=\bigcap_{\delta>0} \operatorname{cl} F\left(t_{0}, x_{0} ; \delta\right) .
$$

We shall say that $F(t, x)$ has property (Q) at $\left(t_{0}, x_{0}\right)$ in $A$ if

$$
F\left(t_{0}, x_{0}\right)=\bigcap_{\delta>0} \operatorname{clco} F\left(t_{0}, x_{0} ; \delta\right) .
$$

We shall say that $F(t, x)$ has properties (U), (Q) in $A$ if $F(t, x)$ has properties $(\mathrm{U}),(\mathrm{Q})$ at every $(t, x)$ in $A$, respectively. A set $F(t, x)$ satisfying property (U) is necessarily closed, and a set satisfying property $(\mathrm{Q})$ is necessarily closed and convex (see Ref. 1, Section 4). If the set $F(t, x)$ is closed for each $(t, x)$ in $A$ and is an upper semicontinuous function in $A$, then $F(t, x)$ has property $(\mathrm{U})$ in $A$. If the set $F(t, x)$ is closed and convex for every $(t, x)$ in $A$ and $F(t, x)$ is an upper semicontinuous function in $A$, then $F(t, x)$ has property (Q) in $A$ (see Ref. 1, Section 4).

Let $G \subset E_{1}$ have the form $[a, \infty),(-\infty, b]$, or $(-\infty, \infty), a, b$ finite. We shall say that

$$
G=\bigcup_{j=1}^{\infty} G_{j}
$$

is a typical representation of $G$ if each $G_{j}$ is a finite closed interval of $G$, and $G_{j} \subset G_{j+1}, j=1,2, \ldots$ Clearly, there are infinitely many such representations for $G$.

Let

$$
\begin{array}{r}
X=\left\{\alpha(t), \alpha_{1} \leq t \leq \alpha_{2}: \alpha(t) \text { a continuous } n\right. \text {-vector function, } \\
\left.\qquad t \in E_{1}, \alpha_{1}, \alpha_{2} \text { finite }\right\} .
\end{array}
$$


Let $\alpha(t), \alpha_{1} \leq t \leq \alpha_{2}, \beta(t), \beta_{1} \leq t \leq \beta_{2}$, be any two elements of $X$. We define the distance $\rho(\alpha, \beta)$ by first extending $\alpha(t)$ and $\beta(t)$ outside their intervals of definition by constancy and continuity in $(-\infty, \infty)$, and then let

$$
\rho(\alpha, \beta)=\left|\alpha_{1}-\beta_{1}\right|+\left|\alpha_{2}-\beta_{2}\right|+\max _{-\infty<t<\infty}|\alpha(t)-\beta(t)| .
$$

It is known that $X$, under $\rho$, is a complete metric space, and that Ascoli's theorem holds, that is, if $x_{n}$ is a sequence of equicontinuous vector functions of $X$, whose graphs in the $t x$-space are equibounded, then there exists a subsequence of $x_{n}$ which converges in the $\rho$-metric to an element $x$ of $X$.

\section{Closure Theorems}

We shall first discuss a closure theorem for a half-infinite domain of the form $\left[t_{1}, \infty\right), t_{1}$ finite. We shall then discuss domains of the form $\left(-\infty, t_{2}\right)$ and $(-\infty, \infty)$. We shall continue to use the same notations as Sections 2 and 3 .

In order to include the behavior of the cost function $f_{0}$ directly into our system, we shall here augment the control system described in Section 2. Specifically, we let

$$
x=\left(x^{1}, \ldots, x^{n}\right)=(y, z),
$$

where

$$
y=\left(x^{1}, \ldots, x^{s}\right), \quad z=\left(x^{s+1}, \ldots, x^{n}\right) .
$$

We may think of the $y$-vector as corresponding to the state vector $x$ of Section 2, and of the $z$-vector as corresponding to the behaviour of the cost functional, with $n=s+1$. Accordingly, we assume that the state function $f(t, y, u)$ depends only on $x^{1}, \ldots, x^{s}$ and, for a given trajectory $x(t)=(y(t)$, $z(t))$, the vector $y(t)$ possesses a derivative a.e. We impose a different set of assumptions on $z$, the remaining $n-s$ components of $x$.

Theorem 4.1. Closure Theorem. Let $A_{0}$ be a closed subset of the ty-space $E_{1} \times E_{s}$, so that $A=A_{0} \times E_{n-s}$ is a closed subset of the $t x$-space $E_{1} \times E_{n}$. Let $U(t, y)$ denote a closed subset of $E_{m}$ for every $(t, y) \in A_{0}$, let

$$
M_{0}=\left\{(t, y, u):(t, y) \in A_{0}, u \in U(t, y)\right\} \subset E_{1+s+m},
$$

and let

$$
f(t, y, u)=\left(f_{1}(t, y, u), \ldots, f_{n}(t, y, u)\right)
$$

be a continuous vector function from $M_{0}$ into $E_{n}$. Let

$$
Q(t, y)=f(t, y, U(t, y))
$$


be a closed convex subset of $E_{n}$ for every $(t, y) \in A_{0}$. Assume that $U(t, y)$ has property $(\mathrm{U})$ in $A_{0}$ and that $Q(t, y)$ has property $(\mathrm{Q})$ in $A_{0}$. Let the interval $(-\infty, \infty)$ have a typical representation $\cup G_{j}, G_{j} \subset G_{j+1}$, with

$$
G_{j}=\left[g_{1 j}, g_{2 j}\right] \text { and }\left|g_{2 j}-g_{1 j}\right| \leq d_{j}<\infty \text {. }
$$

Let $x_{k}(t), t_{1 k} \leq t \leq t_{2 k}, t_{1 k}$ finite and bounded, $t_{2 k}$ finite or infinite, $k=$ $1,2, \ldots$, be a sequence of trajectories, $x_{k}(t)=\left(y_{k}(t), z_{k}(t)\right), t_{2 k} \rightarrow \infty$ as $k \rightarrow$ $\infty, t_{2 k}$ possibly infinite. Then, there is an integer $q$ so that $t_{1}, t_{1 k} \in G_{q}$. We assume that, within any of the intervals $G_{j}, j \geq q$, the $s$-vector function $y_{k}(t)$ converges in the $\rho$ metric (relative to $G_{j}$ )

$$
\rho\left(y_{k}, y\right)=\left|t_{1}-t_{i k}\right|+\max _{t \in G_{j}}\left|y_{k}(t)-y(t)\right|,
$$

toward a vector function $h(t), t_{1} \leq t<\infty, t_{1}$ finite, which is AC on bounded intervals, and where $y_{k}, y$ are extended in $G_{j}$, if necessary, by constancy and continuity, and $k$ is taken sufficiently large so that $t_{2 k} \geq g_{2 j}$. For this same sequence $x_{k}(t)$, let us also assume that the $(n-s)$-vector function $z_{k}(t)$ converges pointwise a.e. in $t_{1} \leq t<\infty$ toward a vector function $z(t)$ which admits a decomposition

$$
z(t)=Z(t)+S(t)
$$

where $Z(t)$ is an AC function for $t_{1} \leq t<\infty$ and $S^{\prime}(t)=0$ a.e. in $t_{1} \leq t<\infty$, that is, $S(t)$ is a singular function. Then, the vector function

$$
X(t)=[y(t), Z(t)], \quad t_{1} \leq t<\infty,
$$

is an admissible trajectory.

Proof. By assumption, $t_{1}$ is finite. Also, the vector functions

$$
\begin{gathered}
\phi(t)=X^{\prime}(t)=\left(y^{\prime}(t), Z^{\prime}(t)\right), \quad t_{1} \leq t<\infty, \\
\phi_{k}(t)=x_{k}^{\prime}(t)=\left(y_{k}^{\prime}(t), Z_{k}^{\prime}(t)\right)=f\left(t, y_{k}(t), u_{k}(t)\right), \quad t_{1} \leq t<\infty, \quad \\
k=1,2, \ldots,
\end{gathered}
$$

exist a.e. and are L-integrable over bounded time intervals. Moreover, since $y_{k}(t) \rightarrow y(t)$ as $k \rightarrow \infty$, and since $A_{0}$ is closed, it follows that $(t, y(t)) \in A_{0}$ for all $t$ in $\left[t_{1}, \infty\right)$, and hence

or

$$
(t, y(t), Z(t)) \in A_{0} \times E_{n-s},
$$

$$
(t, X(t)) \in A, \quad t_{1} \leq t<\infty .
$$

It remains to show that there is a measurable control $u(t), t_{1} \leq t<\infty$, such that

$$
\phi(t)=X^{\prime}(t)=\left(y^{\prime}(t), Z^{\prime}(t)\right)=f(t, y(t), u(t)), \quad u(t) \in U(t, y(t)),
$$


a.e. in $\left[t_{1}, \infty\right)$. This is done by using the same local argument as used for the bounded time-domain case. More precisely, for a.a. $t, X^{\prime}(t)=\left[y^{\prime}(t), Z^{\prime}(t)\right]$ exists and is finite, $S^{\prime}(t)$ exists and is zero, and $z_{k}(t) \rightarrow z(t)$. Let $t_{0}$ be such a point with $t_{1}<t_{0}<\infty$. By construction, for any $\sigma>0$, there is an integer $r>0$ so that $\left[t_{0}-\sigma, t_{0}+\sigma\right] \subset G_{j}$ for $j \geq r$ and, for some $k_{0}$ and all $k \geq k_{0}$, also $t_{1 k}<t_{0}-\sigma<t_{0}+\sigma<t_{2 k}$. Now, $y_{k}(t)$ converges to $y(t)$ in the $\rho$ metric in $G_{j}$ for $j \geq q$. Hence, $y_{k}(t) \rightarrow y(t)$ uniformly in $\left[t_{0}-\sigma, t_{0}+\sigma\right] \subset G_{j}$ for $k \geq k_{0}$, $j \geq \max (r, q)$. By restricting our attention to the interval $\left[t_{0}-\sigma, t_{0}+\sigma\right]$, one may show, in the same way as done for the bounded domain case (see Ref. 1, Section 5), that $\phi\left(t_{0}\right) \in Q\left(t_{0}, y_{0}\right)$, where $y_{0}=y\left(t_{0}\right)$. That is, there are points $\bar{u}=\bar{u}\left(t_{0}\right) \in U\left(t_{0}, y_{0}\right)$ such that

$$
\phi\left(t_{0}\right)=f\left(t_{0}, y\left(t_{0}\right), \tilde{u}\left(t_{0}\right)\right) .
$$

Since $t_{0}$ was chosen arbitrarily, relation (2) holds a.e. in $\left[t_{1}, \infty\right)$. Hence, there exists at least one function $\bar{u}(t)$, defined a.e. in $\left[t_{1}, \infty\right)$, for which relation (1) holds a.e. By the implicit function theorem of McShane and Warfield (Ref. 10 ), it follows that there is at least one measurable function $u(t)$, for which relation (1) holds a.e. in $\left[t_{1}, \infty\right)$. The closure theorem is thereby proved.

Corollary 4.1. The above closure theorem remains valid if $t_{1 k} \rightarrow t_{1}=$ $-\infty$ and $t_{2 k}$ converges to a finite value $t_{2}$, or if both $t_{1 k} \rightarrow t_{1}=-\infty, t_{2 k} \rightarrow t_{2}=$ $\infty$ (the theorem also remains valid if both $t_{1}$ and $t_{2}$ are finite).

Proof. If $t_{1}=-\infty$, then the proof of the closure theorem is the same as that given, except with $t_{1}, t_{1 k}$ interchanged with $t_{2}, t_{2 k}$. If $t_{1}=-\infty, t_{2}=\infty$, the proof again holds with minor changes. In particular, given any $G_{i}$, convergence in the $\rho$-metric is then replaced by uniform convergence for $k$ sufficiently large. For $t_{1}, t_{2}$ finite, the hypotheses of the closure theorem imply that the assumptions of the corresponding closure theorem for bounded domains are in effect (see Ref. 1, Section 5). The corollary is thereby proved.

\section{Closed Classes of Admissible Pairs}

The definition of admissible pairs was given in Section 2. A class $\Omega$ of admissible pairs will be called closed if, given any sequence $x_{k}(t), u_{k}(t)$, $t_{1 k} \leq t \leq t_{2 k}, k=1,2, \ldots$ ( $t_{1 k}$ or $t_{2 k}$ possibly infinite), of pairs from $\Omega$ which converge in the compact open topology (in the sense described in the closure theorem) toward a function $x(t), t_{1} \leq t \leq t_{2}$, which is a trajectory generated by some admissible control $u(t)$, then $(x(t), u(t)) \in \Omega$. We will need this property in the next section, when we discuss the existence of optimal pairs. 
For the systems of Section 2, closed classes $\Omega$ are often defined in terms of boundary conditions. If the domain of the control system is bounded, then the boundary conditions for an admissible class $\Omega$ may be written as

$$
\left(t_{1}, x\left(t_{1}\right), t_{2}, x\left(t_{2}\right)\right) \in B,
$$

where $B$ is a fixed subset of $E_{2 n+2}$. If we assume that $B$ is closed, then the class $\Omega$ of all admissible pairs satisfying boundary conditions (3) is automatically closed. The notation of (3) can be extended to boundary conditions over an infinite time domain. In particular,

(a) if $t_{1}=-\infty, t_{2}$ finite, then we ask that

$$
\left(x\left(t_{1}\right), t_{2}, x\left(t_{2}\right)\right) \in B^{\prime} \subset E_{2 n+1}
$$

or

$$
\left(t_{1}, x\left(t_{1}\right), t_{2} x\left(t_{2}\right)\right) \in B=\{-\infty\} \times B^{\prime} \subset E_{2 n+2},
$$

where $x\left(t_{1}\right)$, for $t_{1}=-\infty$, denotes $\lim _{t \rightarrow-\infty} x(t)=x(-\infty)$;

(b) if $t_{2}=\infty, t_{1}$ finite, then we ask that

$$
\left(t_{1}, x\left(t_{1}\right), x\left(t_{2}\right)\right) \in B^{\prime} \subset E_{2 n+1}
$$

or

$$
\left(t_{1}, x\left(t_{1}\right), t_{2}, x\left(t_{2}\right)\right) \in B=B^{\prime} \times\{\infty\} \subset E_{2 n+2} ;
$$

(c) if $t_{1}=-\infty, t_{2}=\infty$, then we ask

$$
\left(x\left(t_{1}\right), x\left(t_{2}\right)\right) \in B^{\prime} \subset E_{2 n}
$$

or

$$
\left(t_{1}, x\left(t_{1}\right), t_{2}, x\left(t_{2}\right)\right) \in B=\{-\infty\} \times B^{\prime} \times\{\infty\} \subset E_{2 n+2} .
$$

Hence, with these conventions, we may still write for all cases that $\left(t_{1}, x\left(t_{1}\right), t_{2}, x\left(t_{2}\right)\right) \in B \subset E_{2 n+2}$, that is, (3).

As mentioned, if the domain of a control system is bounded, then it is fairly easy to show that $\Omega$ is closed in many cases. However, for unbounded domains, verification of the boundary conditions is a harder task; in general, we must show that the boundary conditions satisfy property $(P)$ : if $x(t)$, $t_{1} \leq t \leq t_{2}, x_{k}(t), t_{1 k} \leq t \leqq t_{2 k}$, are trajectories for the control system, with $x_{k}$ converging to $x$ in the compact open topology, if $x_{k}$ satisfies the boundary conditions (3), and if $I\left[x_{k}, u_{k}\right] \leq F, k=1,2, \ldots$, for some constant $F$, then $x$ also satisfies boundary conditions (3).

Let us now consider conditions which guarantee that property $(P)$ holds. These conditions, however, may be more restrictive than needed; in 
Section 7 , we consider some examples where these conditions may be relaxed.

Theorem 5.1. Given a control system as described above, let us further assume that

(i) $f_{0}(t, x, u) \geq-\psi(t)$ for all $(t, x, u) \in M$, where $\psi(t) \geq 0$ is L-integrable for $t \in V=\left\{t:(t, x) \in A\right.$ for some $\left.x \in E_{n}\right\}$;

(ii) for any given $0<\epsilon<1$, there is a $\bar{t}=\bar{t}(\epsilon)>0$ so that

$$
|f(t, x, u)| \leq \epsilon\left[f_{0}(t, x, u)+\psi(t)\right]
$$

for all $(t, x, u) \in M$ with $t \geq \bar{t}$.

Then, the control system satisfies property (P).

Proof. Let $x_{k}(t), t_{1 k} \leq t \leq t_{2 k}\left(t_{1 k}, t_{2 k}\right.$ finite or infinite), converge to $x(t), t_{1} \leq t \leq t_{2}\left(t_{1}, t_{2}\right.$ finite or infinite $)$, in the compact open topology, with

$$
\left|\int_{t_{1 k}}^{t_{2 k}} f_{0}\left(t, x_{k}(t), u_{k}(t)\right) d t\right| \leq F
$$

$K=1,2, \ldots$ For convenience, we assume that $t_{1}=-\infty, t_{2}=\infty$ (that is, $t_{1 k} \rightarrow-\infty, t_{2 k} \rightarrow \infty$ as $k \rightarrow \infty$ ); the case for $t_{1}$ or $t_{2}$ finite may easily be handled in the usual way. The boundary conditions (3) for $x(t),-\infty<t<\infty$, thus, become

$$
\left(\lim _{t \rightarrow-\infty} x(t), \lim _{t \rightarrow \infty} x(t)\right) \in B^{\prime} \subset E_{2 n},
$$

$B^{\prime}$ closed. Given $\epsilon>0$, let $t_{a}, t_{b}$ be chosen so that $t_{a}<-\bar{t}$ and $t_{b}>\bar{t}$. Choose $k_{0}=k_{0}\left(t_{a}, t_{b}\right)$, so that $t_{1 k}<t_{a}<-\bar{t}<\bar{t}<t_{b}<t_{2 k}$ for $k>k_{0}$. Then, for any $t^{\prime}=t^{\prime}(\epsilon)$ and $t^{2}=t^{2}(\epsilon)$ with $t_{a}<t^{\prime}<-\bar{t}, \bar{t}<t^{2}<t_{b}$,

$$
\begin{aligned}
\left|\left(x_{k}\left(t^{\prime}\right), x_{k}\left(t^{2}\right)\right)-\left(x_{k}\left(t_{k}\right), x_{k}\left(t_{k}\right)\right)\right| & \leq \int_{t_{1 k}}^{-i}(f) d t+\int_{i}^{t_{2 k}}(f) d t \\
& \leq \epsilon \int_{t_{1 k}}^{-\bar{t}}\left(f_{0}+\psi\right) d t+\epsilon \int_{i}^{t_{2 k}}\left(f_{0}+\psi\right) d t \\
& \leq \epsilon \int_{t_{1 k}}^{t_{2 k}}\left(f_{0}+\psi\right) d t \\
& \leq \epsilon \int_{t_{1 k}}^{t_{2 k}} f_{0} d t+\epsilon \int_{-\infty}^{\infty} \psi d t \leq \epsilon(F+L)=\epsilon^{\prime}
\end{aligned}
$$


for $k>k_{0}$, where

$$
\int_{-\infty}^{\infty} \psi(t) d t=\mathrm{L}<\infty
$$

Since $\left(x_{k}\left(t_{1 k}\right), x_{k}\left(t_{2 k}\right)\right) \in B^{\prime}$ for all $k$, we may conclude that

$$
\left(x_{k}\left(t^{\prime}\right), x_{k}\left(t^{2}\right)\right) \in\left[B^{\prime}\right]_{\epsilon^{\prime}}
$$

for all $t^{\prime}, t^{2}$ with $t_{a}<t^{\prime}<-\bar{t}<\bar{t}<t^{2}<t_{b}$, for $k>k_{0}$, where $\left[B^{\prime}\right]_{\delta}$ denotes the closed $\delta$-neighborhood of $B^{\prime}$. By taking $k \rightarrow \infty$, we thus have $\left(x\left(t^{\prime}\right), x\left(t^{2}\right)\right) \in$ $\left[B^{\prime}\right]_{\epsilon^{\prime}}$ for all $t^{\prime}, t^{2}$ with $t_{a}<t^{\prime}<-\bar{t}<\bar{t}<t^{2}<t_{b}$. Moreover, relation (5) will hold for any $t_{a}, t_{b}$, with $t_{a}<-\bar{t}, t_{b}>\bar{t}$, and $k \geq k_{0}\left(t_{a}, t_{b}\right), k_{0}\left(t_{a}, t_{b}\right)$ suitably chosen. Hence, $\left(x\left(t^{\prime}\right), x\left(t^{2}\right)\right) \in\left[B^{\prime}\right]_{\epsilon^{\prime}}$ for all $t^{\prime}, t^{2}$, with $-\infty<t^{\prime}<-\bar{t}<\bar{t}<t^{2}<$ $\infty$. Since $\epsilon$, and thus $\epsilon^{\prime}$, was chosen arbitrarily small, and since $B^{\prime}$ is closed, it follows that

$$
\left(\lim _{t \rightarrow-\infty} x(t), \lim _{t \rightarrow \infty} x(t)\right) \in B^{\prime} .
$$

Theorem 5.1 is thereby proved.

The following corollary follows immediately from Theorem 5.1.

Corollary 5.1. Let the hypotheses of Theorem 5.1 be in force, with the exception that condition (ii) is replaced with the following condition:

(iii) there is a function $\theta(t) \geq 0$ for $t \in V$, with $\theta(t) \rightarrow 0$ as $t \rightarrow \infty$, so that

$$
\left.|f(t, x, u)| \leq \theta(t)\left[f_{0} t, x, u\right)+\psi(t)\right]
$$

for all $(t, x, u) \in M$.

Then, the control system again satisfies property $(\mathrm{P})$.

Theorem 5.2. Given a control system as described in the beginning of this section, if there is an L-integrable function $\Gamma(t) \geq 0, t \in V=$ $\left\{t:(t, x) \in A\right.$ for some $\left.x \in E_{n}\right\}$, such that

$$
|f(t, x, u)| \leq \Gamma(t)
$$

for all $(t, x, u)$ in $M$, then the control system satisfies property (P).

The proof of this theorem is very similar to that of Theorem 5.1, with $t_{a}$, $t_{b}$ now chosen so that, for a given $\epsilon>0$,

$$
\int_{\infty}^{t_{a}} \Gamma(t) d t \leq \epsilon, \quad \int_{t_{b}}^{\infty} \Gamma(t) d t \leq \epsilon .
$$

Corollary 5.2. Given a control system as described in the beginning of this section, if there are L-integrable functions $\theta(t) \geq 0, \phi(t) \geq 0, t \in V$, and a 
constant $C \geq 0$, so that

$$
\left|f_{0}(t, x, u)\right| \leq \theta(t), \quad|f(t, x, u)| \leq C\left|f_{0}(t, x, u)\right|+\phi(t),
$$

for all $(t, x, u) \in M$, then the control system satisfies property $(\mathrm{P})$.

The corollary follows immediately from Theorem 5.2 with

$$
\Gamma(t)=C \theta(t)+\phi(t) \text {. }
$$

\section{Existence Theorems}

Let us now consider existence theorems for the control systems described in Section 2.

Theorem 6.1. Existence Theorem. Let $A$ be a closed subset of the $t x$-space $E_{1} \times E_{n}$, with the property that, if $G$ is a closed finite interval, then $A_{G}=\left(G \times E_{n}\right) \cap A$ is compact. For every $(t, x) \in A$, let $U(t, x)$ be a closed subset of the $u$-space $E_{m}$. Let

$$
\tilde{f}(t, x, u)=\left(f_{0}(t, x, u), f_{1}(t, x, y), \ldots, f_{n}(t, x, u)\right)=\left(f_{0}(t, x, u), f(t, x, u)\right)
$$

be a continuous vector function on the set

$$
M=\{(t, x, u):(t, x) \in A, u \in U(t, x)\} .
$$

Assume that, for every $(t, x) \in A$, the set

$$
\left.\tilde{Q}(t, x)=\left\{z^{0}, z\right): z^{0} \geq f_{0}(t, x, u), z=f(t, x, u), u \in U(t, x)\right\} \subset E_{1+n}
$$

is convex and closed. Assume that $U(t, x)$ satisfies property (U) in $A$ and $\tilde{Q}(t, x)$ satisfies property $(\mathrm{Q})$ in $A$. Let $E_{1}$ have a typical representation $\cup G_{j}, G_{j} \subset G_{j+1}$, with

$$
G_{j}=\left[g_{1 j}, g_{2 j}\right] \quad \text { and }\left|g_{1 j}-g_{2 j}\right| \leq d_{j}<\infty .
$$

Let us assume that the following growth condition $(\gamma)$ holds:

$(\gamma)$ given any $\epsilon>0$ and any $G_{j}$, there is a function $\Phi_{j \epsilon}(t) \geq 0$ (which may depend on $\epsilon$ and $G_{j}$ ) which is L-integrable for $t \in G_{j}$, such that

$$
|f(t, x, u)| \leq \Phi_{j \epsilon}(t)+\epsilon f_{0}(t, x, u)
$$

for all $(t, x, u) \in M$ with $t \in G_{j}$ (see also Corollary 6.1).

Let us also assume that

$$
f_{0}(t, x, u) \geq \psi(t)
$$

for all $(t, x, u) \in M$, where $\psi(t) \geq 0$ is a given L-integrable function on

$$
V=\left\{t:(t, x) \in A \text { for some } x \in E_{n}\right\} .
$$


Then, the cost functional $I[x, u]$ has an absolute minimum in any nonempty closed class $\Omega$ of admissible pairs. The criteria for the closedness of classes $\Omega$ can be replaced by property (P); see Corollary 6.2.

We shall prove this theorem under the assumption that the domain of the control system is $-\infty<t<\infty$. This is the most interesting and most difficult case, since bounded domains may be treated as in Refs. 1 or 4 .

Remark 6.1. From the hypotheses, it may be shown that

$$
i=\inf _{\Omega} I[x, u]
$$

is finite (this shall be done below). Hence, we may consider a minimizing sequence $x_{k}(t), u_{k}(t), t_{1 k} \leq t \leq t_{2 k}$ ( $t_{1 k}$ and $t_{2 k}$ finite or infinite), $k=1,2, \ldots$, such that $I\left[x_{k}, u_{k}\right] \rightarrow i$ as $k \rightarrow \infty$. From this point, the argument differs from the usual one for bounded domains, as presented by Cesari in Refs. 1 or 4 , only if $t_{1 k} \rightarrow-\infty$ or $t_{2 k} \rightarrow \infty$ or both. The argument for $t_{1 k} \rightarrow-\infty$ is somewhat different from the argument for $t_{k} \rightarrow \infty$. Moreover, if one of these limits is finite, this endpoint can be treated by easily modifying the argument for $t_{1 k} \rightarrow-\infty, t_{2 k} \rightarrow \infty$, along the lines of Ref. 1, Section 7 (using the $\rho$-metric). Hence, we shall assume that $t_{1 k} \rightarrow-\infty$ and $t_{2 k} \rightarrow \infty$ as $k \rightarrow \infty$. Consequently, for any $G_{j}, k$ may then be chosen sufficiently large so that $\left[t_{1 k}, t_{2 k}\right] \supset G_{j}$. Hence, we may assume that the convergence of $x_{k}$ in $G_{j}$ is uniform, and thus, we essentially lose nothing if we assume that the domain of the control system is $-\infty<t<\infty$. For these reasons, we shall prove the existence theorem for the fixed unbounded domain $-\infty<t<\infty$ (again, the other cases may be obtained by modifying the following proof along the lines of Ref. 1 or Ref. 4).

Proof. By hypothesis, $f_{0}(t, x, u) \geq \psi(t)$ for all $(t, x, u) \in M$, where $\psi(t)$ is L-integrable in $-\infty<t<\infty$. Let

$$
\int_{-\infty}^{t} \psi(t) d t=L(t) \quad \text { and } \int_{-\infty}^{\infty} \psi(t) d t=L .
$$

Then, for every pair $x(t), u(t),-\infty<t<\infty$, of $\Omega$, we have

$$
I[x, u]=\int_{-\infty}^{\infty} f_{0} d t \geq-\int_{-\infty}^{\infty} \psi(t) d t \geq-L>-\infty .
$$

Let

$$
i=\inf _{(x, u) \in \Omega} I[X, u] .
$$

Then, by (9) and $\Omega \neq \phi, i$ is finite. 
Let $x_{k}(t), u_{k}(t),-\infty<t<\infty, k=1,2, \ldots$, be a sequence of admissible pairs in $\Omega$, such that $I\left[x_{k}, u_{k}\right] \rightarrow i$ as $k \rightarrow \infty$. We may assume, for convenience,

$$
i \leq I\left[x_{k}, u_{k}\right]=\int_{-\infty}^{\infty} f_{0}\left(t, x_{k}(t), u_{k}(t)\right) d t<i+k^{-1} \leq i+1,
$$

$k=1,2, \ldots$ Let $E_{1}$ have a typical representation $\cup G_{j}$ as described in the hypotheses. By assumption,

$$
A_{G_{j}}=\left(G_{j} \times E_{n}\right) \cap A
$$

is compact for any $j$. Hence, for any $j$, the sequence $\left[x_{k}(t)\right], t \in G_{j}$, is equibonded. We now show, as in Ref. 4 , that, for any given $j$, the AC vector functions $x_{k}(t), t \in G_{j}$, are equiabsolutely continuous. Let us fix $j$. Let $\epsilon>0$ be given, and set

$$
\sigma=2^{-1}(i+1+L)^{-1} \epsilon, \quad 0<\sigma<\infty .
$$

Since $\Phi_{j \sigma}(t)$ is L-integrable on $G_{j}$, there exists an $\eta>0$ such that

$$
\int_{E} \Phi_{j \sigma}(t) d t \leq \epsilon / 2
$$

for every measurable subset $E$ of $G_{j}$ with meas $E<\eta$. Hence, for any such set $E$, we have

$$
\begin{aligned}
\int_{E}\left(x_{k}^{\prime}(t)\right) d t & =\int_{E}\left(f\left(t, x_{k}(t), u_{k}(t)\right) d t \leq \int_{E}\left[\Phi_{j \sigma}(t)+\sigma f_{0}\left(t, x_{k}(t), u_{k}(t)\right)\right] d t\right. \\
& \leq \int_{E} \Phi_{j \sigma} d t+\sigma \int_{E}\left[f_{0}+\psi\right] d t \leq \int_{E} \Phi_{j \sigma} d t+\sigma \int_{-\infty}^{\infty}\left[f_{0}+\psi\right] d t \\
& \leq \epsilon / 2+\sigma(i+1+L)=\epsilon / 2+\epsilon / 2=\epsilon,
\end{aligned}
$$

$k=1,2, \ldots$. Hence, the functions $x_{k}(t), t \in G_{j}, k=1,2, \ldots$, are equiabsolutely continuous. Since $j$ was chosen arbitrarily, this property holds for all $G_{j}$.

Let us now consider the sequence of AC scalar functions $x_{k}^{o}(t)$ defined by

$$
x_{k}^{0}(t)=\int_{-\infty}^{t} f_{0}\left(s, x_{k}(s), u_{k}(s), u_{k}(s)\right) d s, \quad-\infty<t<\infty
$$

with

$$
\begin{aligned}
\lim _{t \rightarrow \infty} x_{k}^{0}(t) & =I\left[x_{k}, u_{k}\right] \rightarrow i \quad \text { as } k \rightarrow \infty, \\
i & =\lim _{t \rightarrow \infty} x_{k}^{0}(t)<i+k^{-1} \leq i+1 .
\end{aligned}
$$


Let

$$
u_{k}^{0}(t)=f_{0}\left(t, x_{k}(t), u_{k}(t)\right),-\infty<t<\infty
$$

and let

$$
u^{-}(t)=-\psi(t), \quad u_{k}^{+}(t)=u_{k}(t)+\psi(t), \quad-\infty<t<\infty .
$$

Then,

$$
u^{-}(t) \leq 0, \quad u_{k}^{+}(t) \geq 0 \quad \text { a.e. in }-\infty<t<\infty .
$$

Let us define

$$
\begin{aligned}
& y^{-}(t)=\int_{-\infty}^{t} u^{-}(s) d s=-L(t), \quad y_{k}^{+}(t)=\int_{-\infty}^{t} u_{k}^{+}(s) d s, \\
&-\infty<t<\infty, \quad k=1,2, \ldots
\end{aligned}
$$

The function $y^{-}(t)$ does not depend on $k$, and the functions $y_{k}^{+}(t)$ are nonnegative, nondecreasing, and uniformly bounded, since

$$
\begin{aligned}
0 \leq \lim _{t \rightarrow \infty} y_{k}^{+}(t) & =\lim _{t \rightarrow \infty}\left[\left(y_{k}^{+}(t)+y^{-}(t)\right)-y^{-}(t)\right] \\
& =\lim _{t \rightarrow \infty}\left[x_{k}^{0}(t)-y^{-}(t)\right] \leq i+1+L<\infty .
\end{aligned}
$$

Given any $G_{j}$, we may extract, by Ascoli's theorem, a sequence for which $x_{k}(t), t \in G_{j}$, converges uniformly toward a vector function $x(t)$ which is $\mathrm{AC}$, since the functions $x_{k}(t)$ are AC. Since $(-\infty, \infty)$ is a countable union of the $G_{j}$, it follows that a subsequence may be obtained by the diagonal process so that, for any fixed $G_{j}, x_{k}(t), t \in G_{j}$, converges uniformly toward $x(t), t \in G_{j}$, with $x(t) \mathrm{AC},-\infty<t<\infty$. Furthermore, we may now apply Helly's theorem to the sequence $y_{h}^{+}(t)$ to obtain a sequence $y_{k}^{+}(t)$ which converges for every $-\infty<t<\infty$ to a function $Y_{0}^{+}(t),-\infty<t<\infty$, which is nonnegative, nondecreasing, but not necessarily continuous, with

$$
0 \leq Y_{0}^{+}(t) \leq L+i+1, \quad-\infty<t<\infty .
$$

Since $Y_{0}^{+}(t)$ is nondecreasing and bounded, $\lim _{t \rightarrow \infty} Y_{0}^{+}(t)$ exists.

We can now decompose $Y_{0}^{+}(t)$ uniquely into

$$
Y_{0}^{+}(t)=Y^{+}(t)+Z(t), \quad-\infty<t<\infty,
$$

where both $Y^{+}(t), Z(t)$ are nonnegative, nondecreasing, where $Y^{+}(t)$ is AC with

$$
\lim _{t \rightarrow-\infty} Y^{+}(t)=0
$$

and $Z^{\prime}(t)=0$ a.e. in $-\infty<t<\infty$. Let us set

$$
Y(t)=y^{-}(t)+Y^{+}(t) \text {. }
$$


Then, $x_{k}^{0}(t),-\infty<t<\infty$, converges for all $t$ toward $Y(t)+Z(t)$, where $Y(t)$ is a scalar AC function, $-L \leq Y(t) \leq L+i+1$. By construction,

$$
\lim _{t \rightarrow-\infty} Y(t)=\lim _{t \rightarrow-\infty} y^{-}(t)+\lim _{t \rightarrow-\infty} Y^{+}(t)=0 .
$$

Moreover, since

$$
\lim _{t \rightarrow \infty} y^{-}(t)=-L
$$

and $\lim _{t \rightarrow \infty} Y^{+}(t)$ exist, so does $\lim _{t \rightarrow \infty} Y(t)$. We now show that

$$
\lim _{t \rightarrow \infty} Y(t) \leq i \text {. }
$$

For the subsequence $[k]$ we extracted last, we have

$$
\lim _{t \rightarrow \infty} x_{k}^{0}(t)<i+k^{-1}, \quad x_{k}^{0}(t)=y^{-}(t)+y_{k}^{+}(t), \quad-\infty<t<\infty .
$$

Let $\bar{t}<t_{1}<t_{2}<\cdots<t_{m}<t_{m+1}<\cdots$, where

$$
\lim _{t \rightarrow \infty} t_{m}=\infty
$$

Then, since $y_{k}^{+}(t)$ is nondecreasing,

$$
y_{k}^{+}(\tilde{t}) \leq y_{k}^{+}\left(t_{m}\right), \quad m=1,2, \ldots,
$$

and

$$
\begin{aligned}
y^{-}(\bar{t})+y_{k}^{+}(\bar{t}) & \leq y^{-}(\bar{t})+y_{k}^{+}\left(t_{m}\right) \leq y^{-}\left(t_{m}\right)+y_{k}^{+}\left(t_{m}\right)+\left|y^{-}(\bar{t})-y^{-}\left(t_{m}\right)\right| \leq x_{k}\left(t_{m}\right) \\
& =\int_{\bar{i}}^{t_{m}} \psi(s) d s,
\end{aligned}
$$

for $m=1,2, \ldots$ Hence,

$$
y^{-}(\bar{t})+y_{k}^{+}(\bar{t})<i+k^{-1}+\int_{\bar{t}}^{\infty} \psi(s) d s=i+k^{-j}+[L-L(\bar{t})] .
$$

Thus, as $k \rightarrow \infty$ (along the extracted sequence, we have

$$
y^{-}(\bar{t}) \leq i+[L-L(\bar{t})]
$$

or

$$
y^{-}(\bar{t})+Y^{+}(\bar{t})+Z(\bar{t}) \leq i+[L-L(\bar{t})],
$$

where $Z(\bar{t}) \geq 0$. Thus,

$$
Y(\bar{t})=y^{-}(\bar{t})+y^{+}(\bar{t}) \leq i+[L-L(\bar{t})] .
$$

As $\bar{t} \rightarrow \infty$, we thus obtain relation (11). 
In order to apply the closure theorem, we shall consider the following auxiliary problem. Let

$$
\tilde{u}=\left(u^{0}, u\right)=\left(u^{0}, u^{1}, \ldots, u^{m}\right)
$$

let

$$
\left.\tilde{U}(t, x)=\left\{\tilde{u}=u^{0}, u\right): u^{0} \geq f_{0}(t, x, u), u \in U(t, x)\right\}
$$

let

$$
\tilde{x}=\left(x^{0}, x\right)=\left(x^{0}, x^{1}, \ldots, x^{n}\right),
$$

let

$$
\tilde{\tilde{f}}=\tilde{f}(t, x, u)=\left(\tilde{f}_{0}(t, x, u), f(t, x, u)\right)=\left(\tilde{f}_{0}(t, x, u), f_{1}(t, x, u), \ldots, f_{n}(t, x, u)\right),
$$

with $\tilde{f}_{0}=u^{0}$. Thus, $\tilde{\tilde{f}}$ depends only on $t, x, \tilde{u}$ (instead of $t, \tilde{x}, \tilde{u}$ ), and $\tilde{U}$ depends only on $t, x$ (instead of $t, \tilde{x}$ ). The differential system is given by

$$
d \tilde{x} / d t=\tilde{f}(t, x, u)
$$

or

$$
d x^{0} / d t=u^{0}(t), \quad d x^{i} / d t=f_{i}(t, x, u), \quad i=1, \ldots, n,
$$

a.e. in $-\infty<t<\infty$, with constraints

$$
\tilde{u}(t) \in \tilde{U}(t, x(t))
$$

or

$$
u^{0}(t) \geq f_{0}(t, x(t), u(t)), \quad u(t) \in(t, x(t))
$$

a.e. in $-\infty<t<\infty$, with

$$
\lim _{t \rightarrow-\infty} x^{0}(t)=0
$$

and $(x, u) \in \Omega$. We may now apply the closure theorem with $\tilde{x}$ replacing $x, x$ replacing $y, x^{0}$ replacing $z, n+1$ replacing $n, n$ replacing $s$, and $(n+1)-n=$ 1 replacing $n-s$. In addition, the set

$$
\tilde{Q}(t, x)=\tilde{f}(t, x, \tilde{U}(t, x))=\left\{t=\left(t^{0}, z\right): z^{0}=u^{0} \geq\left(f_{0}(t, x, u), z=f(t, x, u)\right\}\right.
$$

replaces $Q(t, x)$, and $\tilde{U}$ replaces $U$. For this new auxiliary problem, the cost functional is

$$
J|\tilde{x}, \tilde{u}|=\int_{-\infty}^{\infty} \tilde{f}_{0} d t=\int_{-\infty}^{\infty} u^{0}(t) d t=\lim _{t \rightarrow \infty} x^{0}(t)
$$


Let us now consider the sequence of trajectories

$$
\tilde{x}_{k}^{0}(t)=\left[x_{k}^{0}(t), x_{k}(t)\right]
$$

and the sequence of corresponding control functions

$$
\tilde{u}_{k}(t)=\left[u_{k}^{0}(t), u_{k}(t)\right]
$$

obtained above, but now relative to the new auxiliary problem. Since

$$
u_{k}^{0}(t)=f_{0}\left(t, x_{k}(t), u_{k}(t)\right), \quad u_{k}(t) \in U(t, x),
$$

we have

$$
\tilde{u}_{k}(t) \in \tilde{U}\left(t, x_{k}(t)\right), \quad-\infty<t<\infty
$$

If we set

$$
x^{0}(t)=Y(t)+Z(t)
$$

we have shown that, given any $G_{j}$, the sequence $\left[x_{k}(t)\right], t \in G_{j}$, converges uniformly to the AC function $x(t)$, while $x_{k}^{0}(t) \rightarrow x^{0}(t)$ as $k \rightarrow \infty$ for all $-\infty<t<\infty$, where $Y(t)$ is AC in $-\infty<t<\infty$ and $Z^{\prime}(t)=0$ a.e. in $-\infty<t<$ $\infty$.

By the closure theorem, we can thus conclude that $X(t)=[Y(t), x(t)]$ is a trajectory for the problem. That is, there is a measurable control function $\tilde{u}(t),-\infty<t<\infty, \tilde{u}(t)=\left(u^{0}(t), u(t)\right)$, with

$$
\begin{gathered}
d Y / d t=u^{0}(t) \geq f_{0}(t, x(t), u(t)), \quad u(t) \in U(t, x(t)), \\
d x / d t=f(t, x(t), u(t)),
\end{gathered}
$$

a.e. in $-\infty<t<\infty$; and, by relation (11),

$$
i \geq \lim _{t \rightarrow \infty} Y(t)=J[\tilde{x}, \tilde{u}]=\int_{-\infty}^{\infty} u^{0}(t) d t .
$$

Moreover, $[x(t), u(t)],-\infty<t<\infty$, is admissible for the original problem, and hence is in $\Omega$, since $\Omega$ is closed by hypothesis. Thus, relations (12) and (13) yield

$$
i \leq I[x, y]=\int_{-\infty}^{\infty} f_{0}(t, x(t), u(t)) d t \leq \int_{-\infty}^{\infty} u^{0}(t) d t \leq i
$$

and hence $I[x, u]=i$, that is, $i$ is attained in $\Omega$. The existence theorem is thereby proved for the fixed domain $-\infty<t<\infty$. As noted before, the other domains may be handled by modifying this proof along the lines of Ref. 1 or Ref. 4. 
Remark 6.2. In the existence theorem, the set $\tilde{Q}(t, x)$ can be shown to have property (Q) in $A$ if $f_{0}$ and $f$ satisfy a growth condition slightly stronger than $(\gamma)$, namely the following:

Given $\epsilon>0$, there exists an $N \geq 0$ such that, for every $(t, x, u) \in M$, with $|u| \geq N$, we have

See Ref. 3.

$$
|f(t, x, u)| \leq \epsilon f_{0}(t, x, u), \quad 1 \leq \epsilon f_{0}(t, x, u) .
$$

The hypotheses of the existence theorem may be modified in certain ways, as is shown in the following corollary.

Corollary 6.1. If, for any given finite interval $G, A_{G}$ is only closed and not necessarily compact, but, if, in addition, we know that (a)

$$
x_{1} f_{1}+\cdots+x_{n} f_{n} \leq F\left[(x)^{2}+1\right]
$$

for all $(t, x, u) \in M$ with $(t, x) \in A_{G}$, and some constant $F \geq 0$ (which may depend on $G$ ), and (b) each trajectory in $\Omega$ goes through at least one point $\left(t^{*}, x\left(t^{*}\right)\right)$ on a given compact subset $\boldsymbol{P}$ of $A$, then the existence theorem still holds. Moreover, condition (a) can be replaced by the hypothesis: $\left(a^{\prime}\right)$ for $(t, x, u) \in M$, and $(t, x) \in A_{G}$, there are constants $K>0, H \geq 0$ (possibly depending on $G$ ) such that

$$
f_{0}(t, x, u) \geq K|f(t, x, u)| \quad \text { for }|x| \geq H .
$$

The growth condition $(\gamma)$ will hold if the following (stronger) growth condition $\left(\gamma^{\prime}\right)$ holds:

$\left(\gamma^{\prime}\right)$ for every compact subset $A_{0}$ of $A$, there is a continuous scalar function $\Phi_{0}(z), 0 \leq z<\infty$, such that $\Phi_{0}(z) / z \rightarrow \infty$ as $z \rightarrow \infty$, and there are two constants $C_{0}, D_{0} \geq 0$, so that $f_{0} \geq \Phi_{0}(|u|),|f| \leq C_{0}+D_{0}|u|$ for all $(t, x, u) \in M$ with $(t, x) \in A_{0}$ (and where $\Phi_{0}, C_{0}, D_{0}$ may depend on $A_{0}$ ).

In addition, it can then be shown that the set $\tilde{Q}(t, x)$, assumed to be convex, automatically satisfies property (Q).

The proof of this corollary is the same as that of the existence theorem, with the modifications described in Ref. 1, Section 7, and Ref. 4.

As noted in the existence theorem, the closedness of classes $\Omega$ may be replaced by property $(\mathrm{P})$, as seen in the following corollary.

Corollary 6.2. Let the assumptions of the existence theorem be in force, except let $\Omega$ be the class of all admissible pairs for this system (with $\Omega$ not assumed closed). If relations (4), (6), (7), or (8) hold, then $\Omega$ satisfies property $(\mathrm{P})$, so that the existence theorem remains in effect for this system. Moreover, if either (7) or (8) hold, then growth condition $(\gamma)$ is automatically satisfied. 
Proof. In the proof of the existence theorem, we used the closedness of $\Omega$ to conclude that $x$, the limit of the minimizing sequence $\left[x_{k}\right]$, was in $\Omega$. However, since $\left[x_{k}\right]$ is a minimizing sequence, we have that $I\left[x_{k}, u_{k}\right]$ is bounded for all $k$ [see relation (10)]; hence, by the theorems and corollaries of Section 5, property (P) holds, and thus $x \in \Omega$. Moreover, if (7) holds, then condition $(\gamma)$ is satisfied with

$$
\Phi_{j \epsilon}(t)=\Gamma(t)+\epsilon \psi(t)
$$

since then

$$
\Phi_{j \epsilon}+\epsilon f_{0}=\Gamma+\epsilon\left(\psi+f_{0}\right) \geq \Gamma \geq|f| .
$$

Similarly, for (8), with

$$
\Gamma(t)=C \theta(t)+\phi(t) .
$$

Corollary 6.2 is thereby proved.

As remarked before, in Section 5, the hypotheses of Corollary 6.2 may be more restrictive than needed; in general, it may be better to treat different boundary conditions separately. In the next section, we shall illustrate some of these differences.

\section{Examples}

Example 7.1. Search Problem, Ref. 11. We wish to determine $u(t)$, $t_{1} \leq t \leq \infty, u(t)$ measurable, so as to minimize

$$
\int_{t_{\mathrm{i}}}^{\infty} p(t) \exp [-u(t)] d t
$$

subject to

$$
\int_{t_{1}}^{\infty} u(t) d t \leq C, \quad 0 \leq u(t) \leq l(t),
$$

$u \in E_{1}, t_{1} \leq t<\infty$. Here, $p(t)$ is a continuous probability density, that is, $p(t)$ is a real-valued nonnegative continuous function with

$$
\int_{t_{1}}^{\infty} p(t) d t=1
$$

$C>0$ is a given constant, and $l(t)$ is a real-valued nonnegative continuous function, which is L-integrable on any bounded interval of $t_{1} \leq t<\infty$.

Let us rewrite this problem by introducing an auxiliary variable $x \in E_{1}$ by setting

$$
d x / d t=u, \quad x\left(t_{1}\right)=0,
$$


so that

$$
\int_{t_{1}}^{\infty} u(t) d t=\lim _{t \rightarrow \infty} x(t) .
$$

Note that, for $t \geq t_{1},|x(t)| \leq L(t)$, where

$$
L(t)=\int_{t_{1}}^{t} f(t) d t<\infty,
$$

so that, without loss of generality, we may require that

$$
\left.(t, x) \in\{t, x): t \geq t_{1}, 0 \leq x \leq L(t)\right\} .
$$

With these new notations, we can rewrite the above problem as: determine $u(t), t_{1} \leq t<\infty, u(t)$ measurable, so as to minimize

$$
\int_{t_{1}}^{\infty} p(t) \exp [-u(t)] d t
$$

with state equation

$$
d x / d t=u
$$

a.e. in $t_{1} \leq t<\infty$, constraints

$$
\begin{gathered}
(t, x) \in A=\left\{(t, x): t \geq t_{1}, 0 \leq x \leq L(t)\right\} \\
u(t) \in U(t)=\{u: 0 \leq u \leq l(t)\}
\end{gathered}
$$

and boundary conditions

$$
x\left(t_{1}\right)=0, \quad \lim _{t \rightarrow \infty} x(t) \leq C .
$$

Let us now show that this problem satisfies the hypotheses of the existence theorem, and hence has a solution. The constraint set $A$ is closed; and, since $L(t)$ is finite for each $t, A_{G}$ is compact for any closed, finite integral $G$ of $t_{1} \leq t<\infty$. Since $l(t)$ is continuous, the control set $U(t)$ is closed and upper semicontinuous in $t_{1} \leq t<\infty$, and hence satisfies property (U) (see Section 3). The set

$$
\begin{aligned}
\tilde{Q}(t, x) & =\tilde{Q}(t)=\left\{\left(z^{0}, z\right): z^{0} \geq p(t) \exp (-u), z=u, 0 \leq u \leq l(t)\right\} \\
& =\left\{\left(z^{0}, z\right): z^{0} \geq p(t) \exp (-z), 0 \leq z \leq l(t)\right\}
\end{aligned}
$$

is closed and convex for each $t$, since $p(t) \exp (-z)$ is a convex function of $z$. From the continuity of $p(t)$ and $l(t)$, it can also be shown that $\tilde{Q}(t)$ is an upper semicontinuous function of $t$ in $t_{1} \leq t<\infty$. Hence, $\tilde{Q}(t)$ has property $(Q)$ in $t_{1} \leq t<\infty$ (see Section 3). 
By the hypotheses on $l(t)$, growth condition $(\gamma)$ is satisfied, and $f_{0}=$ $p(t) \exp (-u) \geq 0$ for all $t$ and $u$. In addition, the class $\Omega$ of all admissible pairs has property $(P)$ and thus is closed. Note that each of the relations (4), (6), (7), (8) fails to hold. For, if $x_{k}, k=1,2, \ldots$, is a sequence of admissible trajectories converging in the compact open topology, then

$$
\lim _{t \rightarrow \infty} x_{k}(t) \leq C, \quad k=1,2, \ldots ;
$$

hence, since

$$
d x_{k} / d t=u \geq 0
$$

for all $k$, we have that $x_{k}(t) \leq C, t_{1} \leq t<\infty$, and thus $x(t) \leq C, t_{1} \leq t<\infty$; in particular,

$$
\lim _{t \rightarrow \infty} x(t) \leq C .
$$

Since $x_{k}\left(t_{1}\right)=0$ for all $k$, we also have $x\left(t_{1}\right)=0$. Thus, $x(t)$ satisfies all the boundary conditions, so that property $(\mathrm{P})$ holds. Finally, $u(t) \equiv 0, x(t) \equiv 0$, $t_{1} \leq t<\infty$, is an admissible pair, and thus $\Omega$ is nonempty. Hence, all the hypotheses of the existence theorem are satisfied, and thus an optimal solution exists for this problem. In the next example, we consider an extension of this problem.

Example 7.2. Search Problem, Ref. 12. We wish to determine $u(t)$, $t_{1} \leq t<\infty, u(t)$ measurable, so as to minimize

$$
\int_{t_{1}}^{\infty} f_{0}(t, u(t)) d t
$$

subject to

$$
\int_{t_{1}}^{\infty} f(t, u(t)) d t \leq C
$$

with $C>0$ a given constant, and $u(t) \in U(t)<E_{1}, U(t)$ closed and satisfying property $(\mathrm{U})$ in $t_{1} \leq t<\infty, f_{0}, f$ continuous, real-valued functions for

$$
(t, u) \in W=\left\{(t, u): t_{1} \leq t<\infty, u(t) \in U(t)\right\},
$$

and $f_{0}(t, u) \geq-\psi(t)$ for all $(t, u) \in W$, where $\psi(t) \geq 0$ is L-integrable for $t_{1} \leq t<\infty$. As in Example 7.1, we introduce the auxiliary variable $x \in E_{1}$ by setting

$$
d x / d t=f(t, u), \quad x\left(t_{1}\right)=0,
$$


so that

$$
\int_{t_{1}}^{\infty} f(t, u) d t=\lim _{t \rightarrow \infty} x(t)
$$

We can now write the control problem as follows: determine $u(t), t_{1} \leq t<\infty$, $u(t)$ measurable, so as to minimize

$$
\int_{t_{1}}^{\infty} f_{0}(t, u(t)) d t
$$

with state equation

$$
d x / d t=f(t, u)
$$

a.e. in $t_{1} \leq t<\infty$, constraints $u(t) \in U(t)$, and boundary conditions $x\left(t_{1}\right)=0$, $\lim _{t \rightarrow \infty} x(t) \leq C$.

In order to apply the existence theorem, we assume that

$$
\tilde{Q}(t, x)=\tilde{Q}(t)=\left\{\tilde{z}=\left(z^{0}, z\right): z^{0} \geq f_{0}(t, u), z=f(t, u), u \in U(t)\right\}
$$

is a convex subset of $E_{2}$. This is the case if, as in Example 7.1, $f$ is linear in $u$ and $f_{0}$ is convex in $u$ for each fixed $t$. We also assume that $\tilde{Q}(t)$ has property (Q). This can be shown to occur in a number of instances, as in Example 7.1 (see Sections 3 and 6; see Refs. 1 and 4).

Additional assumptions are also needed, depending on the control set $U(t)$. In particular, if $U(t)$ is bounded for $t$ in $G, G$ any bounded subinterval of $t_{1} \leq t<\infty$, then, for

$$
(t, u) \in W_{G}=\left(G \times E_{1}\right) \cap W,
$$

$f_{0}$ and $f$ assume values in a compact set in $E_{1}$. Hence, if

$$
\begin{aligned}
A=\left\{(t, x): t \geq t_{1},-\infty<\left(t_{1}-t\right)\left|\left(\min _{(s, u) \in W_{[1, t]}} f(s, u)\right)\right| \leq x\right. \\
\quad \leq\left(t-t_{1}\right)\left|\left(\max _{(s, u) \in W_{[t 1, t]}} f(s, u)\right)\right|<\infty,
\end{aligned}
$$

then, without loss of generality, we can require that $(t, x) \in A$ (as in Example 7.1 ), and it follows that $A_{G}$ is compact, and growth condition (8) is satisfied. Hence, from the existence theorem, if the class $\Omega$ of all admissible pairs is nonempty and closed [or satisfies property $(P)$ ], then at least one optimal pair exists for this problem. If $U(t)$ is not bounded for $t$ in finite subintervals of $t_{1} \leq t<\infty$, then we may assume that $A_{G}$ is compact for each finite subinterval $G$, and the growth condition ( 8 ) holds, or we may assume that the conditions of Corollary 6.1 hold. For example, we may assume that, for any finite interval $G$ of $E_{1}$, there is a continuous function $\Phi(z)$ such that 
$\Phi(z) / z \rightarrow \infty$ as $z \rightarrow \infty$ and

$$
f_{0}(t, u) \geq \Phi(|u|),|f(t, u)| \leq a+b|u|
$$

for $t_{1} \leqq t<\infty, u(t) \in U(t)$, and further there are constants $G>0, H \geqq 0$, such that

$$
f_{0}(t, u) \geqq G|f(t, u)|
$$

for all $t_{1} \leq t<\infty, u(t) \in U(t)$, with $|x| \geq H$. In either case, we may again conclude that, if $\Omega \neq \phi$ and closed, then an optimal solution exists to this problem.

The question of controllability of these systems may be fairly easy to answer. For instance, as in Example 7.1, if $f(t, u)=0$ for $u=0$ (as may occur if $f$ is a cost density associated with $u$ ), then $u(t) \equiv 0$ is admissible if $f_{0}(t, 0)$ is L-integrable, in which case $\Omega \neq \phi$. By Corollary 6.2, we may further guarantee that $\Omega$ satisfies property $(\mathrm{P})$, and hence is closed, if we assume that relation (4), (6), (7), or (8) holds. However, as in Example 7.1, this assumption can be relaxed if $f$ is nonnegative in $W$ (which again is the case if $f$ is a cost density). For, using the same argument as in Example 7.1, it follows that property $(\mathrm{P})$ will hold for this system with no further assumptions needed. If the time domain for this problem is $\left[t_{1}, t_{2}\right]$, a finite interval, then the existence theorems of Cesari (Refs. 1 and 4) can be directly applied to such systems as rewritten above.

Example 7.3. Let us consider Example 7.2, but with $t_{1}=-\infty$. Then, the previous discussion carries over to this system, with the exception that we replace the boundary condition $x\left(t_{1}\right)=0$ with

$$
\lim _{t \rightarrow-\infty} x(t)=0
$$

Hence, the results of Example 7.2 still hold, with the possible exception of property (P). By Corollary 6.2, property (P) holds for the boundary conditions at $t_{1}=-\infty, t_{2}=\infty$, if relation (4), (6), (7), or (8) holds. Again, if $f \geq 0$ on $W$, then we need only require that these relations hold for $t \geq \bar{t}, \bar{t}$ some finite time. Note that, since

$$
\lim _{t \rightarrow-\infty} x_{k}(y)=0
$$

does not imply $x_{k}(t)=0$ for $t$ sufficiently negative, nor that, for any $\epsilon>0$, there is a $\bar{t}=\bar{t}(\epsilon)$ so that $\left|x_{k}(t)\right| \leq \epsilon$ for $t<\bar{t}, k=1,2, \ldots$, we cannot relax these relations at $t_{1}=-\infty$ as was done in Example 7.2 for

$$
\lim _{t \rightarrow \infty} x(t) \leq C \text {. }
$$

In particular, we see that the boundary conditions at the endpoints $t_{1}=-\infty$ and $t_{2}=\infty$ may have to be treated individually. 
Example 7.4. Modified State Regulator Problem, Ref. 13, Section 9.5. We wish to determine $u(t), 0 \leq t<\infty$, measurable, so as to minimize

$$
I[x, u]=\frac{1}{2} \int_{0}^{\infty}\left[x^{T}(t) Q x(t)+u^{T}(t) R u(t)\right] d t,
$$

where $x^{T}, u^{T}$ denote the transpose of the (column) vectors $x, u$, and $Q, R$ are positive-definite, symmetric matrices, with state equation

$$
d x / d t=C x(t)+D u(t)
$$

and constraints $(t, x) \in A$, a closed subset of $[t \geq 0] \times E_{n}, u(t) \in U(t, x)$, a closed subset of $E_{m}$ satisfying property (U) in $A$, and boundary conditions $x(0)=x_{0}, x_{0}$ fixed.

In order to apply the existence theorem and its corollaries, we note that, since $Q$ and $R$ are positive definite and symmetric, $x^{T} Q x$ and $u^{T} R u$ are nonnegative and convex, with

$$
u^{T} R u \geq k|u|^{2}
$$

for some $k>0$, that is,

$$
u^{T} R u \geq \Phi(|u|)
$$

with $\Phi(z)=k z^{2}$. From this, and the linearity of the state equation, it can be shown that $\tilde{Q}(t, x)$ is convex and satisfies property (Q) in $A$ (see Ref. 1, Section 12). Moreover, the integrand of the cost functional is $\geq-\psi \equiv 0$, an $\mathrm{L}$-integrable function in $0 \leq t<\infty$. In addition, if $A_{0}$ is any compact subset of $A$, then it follows that there are numbers $C_{0}, D_{0} \geq 0$, so that

$$
|C x+D u| \leq C_{0}+D_{0}|u|
$$

for all $(t, x, u) \in M$ with $(t, x) \in A_{0}$. By Corollary 6.1 [hypothesis $(\gamma)$ ], this implies that growth condition $(\gamma)$ holds.

Since there are no requirements on $\lim _{t \rightarrow \infty} x(t)$, we can conclude that the class $\Omega$ of all admissible pairs satisfies property $(\mathrm{P})$. Finally, we must consider the sets $A$ and $U(t, x)$. We may let $U(t, x)=E_{m}$ if, for any finite interval $G, A_{G}$ is assumed compact (as is sometimes done when considering the state regulator problem). Alternately, we may let

$$
A=[t \geq 0] \times E_{n}
$$

if we assume that $U(t, x)$ is bounded in $A$, since then hypothesis (a) of Corollary 6.1 is satisfied. Hence, with these assumptions (and Corollary 6.1), if $\Omega \neq \phi$ (appropriate controllability conditions may be found in Ref. 13, Chapter 9), then an optimal solution exists for this problem. A related problem is given in the next example. 
Example 7.5. State Equation Linear in $u$, Ref. 1, Section 13. We wish to determine $u(t), t_{1} \leq t<\infty$, measurable, so as to minimize

$$
I[x, u]=\int_{t_{1}}^{\infty}\left[g(t, x) \phi(u)+g_{0}(t, x)\right] d t,
$$

with state equation

$$
d x_{i} / d t=\sum_{j=1}^{m} g_{i j}(t, x) u_{j}+g_{i}(t, x), i=1, \ldots, n,
$$

or, in vector form,

$$
d x / d t=H(t, x) u+h(t, x)
$$

with

$$
(t, x) \in A \subset\left[t \geq t_{1}\right] \times E_{n},
$$

$A$ closed,

$$
u(t) \in U(t, x) \subset E_{m},
$$

$U(t, x)$ closed and satisfying property (U) in $A$, and $\phi(u)$ a continuous nonnegative convex function of $u$. Assume that $\phi(u) \geq \Phi(|u|)$ for every $u$, where $\Phi(z), 0 \leq z<\infty$, is a continuous function with $\Phi(z) / z \rightarrow 0$ as $z \rightarrow \infty$. Assume that $g(t, x), g_{0}(t, x), g_{i j}(t, x), g_{i}(t, x)$ are continuous in $A$ and that, for every finite interval $G \subset[t \geq 0]$,

$g \geq \mu>0, \quad g_{0} \geq \mu \geq 0, \quad \sum_{i j}\left|g_{i j}\right| \leq C(t) g, \quad \sum_{i j}\left|g_{i j}\right|+\sum_{i}\left|g_{i}\right| \leq C(t) g_{0}$,

$0 \leq C(t) \leq C$ for some constants $\mu>0, C>0$ (possibly depending on $G$ ), and all $(t, x) \in A_{G}$. Let the class $\Omega$ of all admissible pairs consist of pairs of the form $x(t), u(t), t_{1} \leq t<\infty, x(t) \mathrm{AC}, u(t)$ measurable, satisfying the above requirements, and boundary conditions $x\left(t_{1}\right)=x_{1}, x_{1}$ fixed, $\lim x\left(t_{2}\right) \in$ $B \times E_{n}, B$ closed, with $I[x, u]$ finite. Then, in Ref. 1 , Section 13 , it is shown [using (14)] that conditions $\left(\mathrm{a}^{\prime}\right)$ and $\left(\gamma^{\prime}\right)$ of Corollary 6.1 are satisfied for $t$ in a finite interval of $t_{1} \leq t<\infty$.

Hence, if $\Omega$ is nonempty and closed, we can conclude from Corollary 6.1 that an optimal solution exists. As seen in Example 7.4, we may relax the requirements of (14) if we assume that $U(t, x)$ is a bounded set for $(t, x)$ in $A$. If $B=E_{n}$, no additional conditions are needed to show that $\Omega$ is closed. If $B$ is a proper subset of $E_{n}$, then we may guarantee that $\Omega$ is closed if we assume that $C(t) \rightarrow 0$ as $t \rightarrow \infty$. For, in Ref. 1, Section 13, it is shown that, for each $(t, x) \in A$, we have

$$
|f| \leq C(t) e f_{0}
$$


$e>0$ a constant, independent of $G$. Since $C(t) e \rightarrow 0$ as $t \rightarrow \infty, \Omega$ satisfies property (P) by Corollary 5.1 [similar remarks can be made for $t_{1}=-\infty$, with $C(t) \rightarrow 0$ as $t \rightarrow-\infty]$. Thus, if $\Omega$ is nonempty, Corollary 6.2 guarantees a solution exists for this problem.

Example 7.6. Free Problems. Let us consider the problem of determining $x^{\prime}(t), t_{1} \leq t<\infty, x^{\prime}(t)$ measurable, so as to minimize

$$
I[x]=\int_{t_{1}}^{\infty} f_{0}\left(t_{1}, x(t), x^{\prime}(t)\right) d t
$$

$f_{0}$ continuous, with constraints $(t, x(t)) \in A \subset E_{n}, t_{1}$ finite, and $\left(t_{1}, x\left(t_{1}\right)\right.$, $\left.\lim _{t \rightarrow \infty} x(t)\right) \in B^{\prime}$, a closed subset in $E_{1+2 n}$. This is the usual free problem of the calculus of variations, with infinite terminal time. This system can be obtained from those of Section 2 by setting $m=n, f=u$, and $U(t, x)=E_{m}$, so that the state equation reduces to $d x / d t=u$. Again, closed classes $\Omega$ of functions $x(t)$ can be defined in terms of boundary conditions on $x(t)$, as in Section 5. For these free problems, the existence theorem reduces to the following theorem.

Theorem 7.1. Let $A$ be a fixed subset of the $t_{x}$-space $E_{1} \times E_{n}$, with the property that, if $G$ is any finite closed interval,

$$
A_{G}=\left(G \times E_{n}\right) \cap A
$$

is compact. Let $f_{0}(t, x, u)$ be a continuous function on $M=A \times E_{n}$, with $f_{0}(t, x, u)$ a convex function of $u$ in $E_{n}$ for every fixed $(t, x)$ in $A$, and with $f_{0}(t, x, u) \geq-\psi(t)$ for all $(t, x, u)$ in $M$, where $\psi(t) \geq 0$ is L-integrable for all $t$ such that $(t, x) \in A$ for some $x \in E_{n}$. Let $E_{1}$ have a typical representation $\cup G_{j}, G_{j}>G_{j+1}$, with each $G_{j}$ a finite closed interval, so that, for each $G_{j}$, and $\epsilon>0$, there is a function $\Phi_{j \epsilon}(t) \geq 0$ which is L-integrable for $t \in G_{j}$, such that

$$
|u| \leq \Phi_{j \epsilon}(t)+\epsilon f_{0}(t, x, u)
$$

for $(t, x, u) \in M$ with $t \in G_{j}$. Again, this growth condition holds if, for each $j$,

$$
f_{0}(t, x, u) \geq \Phi_{j}(|u|)
$$

for all $(t, x, u) \in M$, with $t \in G_{j}$, where $\Phi_{j}(z), 0 \leq z<\infty$, is a continuous scalar function with $\Phi_{j}(z) / z \rightarrow \infty$ as $z \rightarrow \infty$. For either of the growth conditions, it can be shown that

$$
\tilde{Q}(t, x)=\left\{\left(z^{0}, z\right): z^{0} \geq f(t, x, z), z \in E_{n}\right\}
$$

is convex and satisfies property (Q) in $A$ (see Ref. 14). Then, the cost functional (15) has an absolute minimum in any nonempty closed class $\Omega$ of admissible pairs. If $A_{G}$ is not compact for finite closed intervals $G$, then a 
solution still exists if

$$
f_{0} \geq C|u|
$$

for all $(t, x, u) \in M$ with $t \in G,|x| \geq D$, where $C>0$ and $D \geq 0$ are suitable constants (possibly depending on $G$ ), and if every trajectory $x(t)$ of $\Omega$ possesses at least one point $\left(t^{*}, x\left(t^{*}\right)\right)$ on a given compact subset $P$ of $A$.

The proof of this statement follows directly from the existence theorem (with Corollary 6.1) and the remarks of Cesari in Ref. 1, Section 11, and Ref. 4. Moreover, if there are no requirements on $x(t)$ at $t=\infty$, then the class $\Omega$ of all admissible pairs for this system is closed. If there are conditions on $x(t)$ at $t=\infty$, then we can guarantee that $\Omega$ is closed if relation $(4),(6),(7)$, or (8) is satisfied with $f=u$ (similar remarks hold for $t_{1}=-\infty$ ). Again, in specific problems, we may wish to treat different boundary conditions indivudually. As mentioned in Section 1, Cinquini (Refs. 5-6), Faedo (Refs. 7-9), and others have examined existence theorems for free problems in which $\Omega$ is assumed closed with respect to convergence in the compact open topology; they have not discussed criteria for boundary conditions at infinity.

\section{References}

1. CESARI, L., Existence Theorems for Weak and Usual Solutions in Lagrange Problems with Unilateral Constraints, I, Transactions of the American Mathematical Society, Vol. 124, pp. 369-412, 1966.

2. CESARI, L., Sobolev Spaces and Multidimensional Lagrange Problems of Optimization, Annali della Scuola Normale Superiore di Pisa, Vol. 22, pp. 193-227, 1968.

3. CESARI, L., LA PALM, J. R., and NishiuRA, T., Remarks on Some Existence Theorems for Optimal Control, Journal of Optimization Theory and Applications, Vol. 3, pp. 296-305, 1969.

4. CeSARI, L., Problems of Optimization, Lecture Notes, University of Michigan, Ann Arbor, Michigan.

5. CinquinI, S., Sopra l'Esistenza dell'Estremo Assoluto per gli Integrali Estesi a Intervalli Infiniti, Rendiconti della Accademia Nazionale dei Lincei, Ser. 8, Vol. 32, pp. 320-325 and 845-851, 1962.

6. Cinquini, S., Una Nuova Estensione dei moderni Metodi del Calcolo delle Variazioni, Annali della Scuola Normale Superiore di Pisa, Ser. 2, Vol. 9, pp. 258-261, 1940.

7. FAEDO, S., Il Calcolo delle Variazioni per gli Integrali Estesi a Intervalli Infiniti, Annali della Scuola Normale Superiore di Pisa, Vol. 7, pp. 91-132, 1953.

8. FAEDO, S., Il Calcolo delle Variazioni per gli Integrali su Intervalli Infiniti, Rendiconti di Matematica Applicata, Vol. 8, pp. 94-125, 1949.

9. FAEDO, S., Il Calcolo delle Variazioni per gli Integrali su un Intervallo Infiniti, Commentationes, Pontificia Academia Scientiarum, Vol. 8, pp. 319-421, 1944. 
10. MCShane, E. J., and WARFIELD, R. B., JR., On Filippov's Implicit Function Lemma, Proceedings of the American Mathematical Society, Vol. 18, pp. 41-47, 1967.

11. KoOPMAN, B. O., The Theory of Search, III. The Optimum Distribution of Effort, Operations Research, Vol. 5, pp. 613-626, 1957.

12. ZAHL, S., An Allocation Problem with Applications to Operations Research and Statistics, Operations Research, Vol. 11, pp. 426-441, 1963.

13. ATHENS, M., and FALB, P. L., Optimal Control, McGraw-Hill Book Company, New York, New York, 1966. 